\title{
Optically induced transparency in a micro-cavity
}

\author{
Yuanlin Zheng ${ }^{1}$, Jianfan Yang ${ }^{2}$, Zhenhua Shen ${ }^{1}$, Jianjun Cao $^{1}$, Xianfeng Chen ${ }^{1}$, Xiaogan Liang ${ }^{3}$ and \\ Wenjie Wan ${ }^{1,2}$
}

Electromagnetically induced transparency has the unique ability to optically control transparency windows with low light in atomic systems. However, its practical applications in quantum physics and information science are limited due to rigid experimental requirements. Here we demonstrate a new mechanism of optically induced transparency in a micro-cavity by introducing a four-wave mixing gain to nonlinearly couple two separated resonances of the micro-cavity in an ambient environment. A signature Fano-like resonance was observed owing to the nonlinear interference of the two coupled resonances. Moreover, we show that the unidirectional gain of the four-wave mixing can lead to the remarkable effect of non-reciprocal transmission at the transparency windows. Optically induced transparency may offer a unique platform for a compact, integrated solution to all-optical and quantum information.

Light: Science \& Applications (2016) 5, e16072; doi:10.1038/lsa.2016.72; published online 6 May 2016

Keywords: Fano resonance; induced transparency; micro-cavity; non-reciprocity

\section{INTRODUCTION}

Electromagnetically induced transparency (EIT) is one of the greatest discoveries to verify the quantum interference nature of atomic systems ${ }^{1}$. It allows the flipping of an optical opaque transmission window to a transparent one under a secondary coherent illumination beam even at the few-photon limit ${ }^{2,3}$, which immediately provides an opportunity for light-matter-light interactions that are in great demand by all-optical and quantum information processing ${ }^{4}$. However, the long-lasting challenge is that direct interactions between photons are prohibited unlike electrons in an electronic system. EIT was first proposed and observed in atomic gases ${ }^{1}$, and has also been observed in solid state systems $s^{5,6}$. Much attention has been drawn to its unique properties, including slowing or even stopping light, alloptical switching and photon storage ${ }^{6-9}$. This provides tremendous opportunities for quantum physics and information science. However, in most circumstances, those atoms must be prepared in gas phase, which requires optical cooling or vapor heating techniques combined with vacuum isolation, making it difficult for chip-scale integrations, which are the major proposed applications. Alternatively, classical analogies that mimic the EIT effect are under active pursuit in various physical systems, including coupled resonators ${ }^{10,11}$, photonic crystals $^{12,13}$ and plasmonic meta-materials ${ }^{14}$. However, most of these rely on linear coupling between the resonances, which can only exhibit EIT-like spectra, but lack of actively controlled transparency. Until recently, successful attempts ${ }^{15-18}$ have cloned the idea of EIT in an opto-mechanical micro-cavity to induce a narrow transparency with the aid of mechanical oscillations that are excited through Brillouin scattering (BS) nonlinearity, which provide enough phonons to couple some hybrid optical-mechanical resonances that are similar to their counterparts, photons in the EIT. However, most of these attempts still require cooling and vacuum systems to preserve the high-quality mechanical modes; furthermore, careful designs must be addressed for both the mechanical and optical modes.

In this work, we were motivated by the EIT in atomic systems. We report an optically induced transparency (OIT) scheme in a compact micro-cavity in an ambient environment by exploring cavityenhanced four-wave mixing (FWM) gain to introduce a transparency window in an opaque resonance dip. This resonance dip directly results from the interference between two resonances that are coupled nonlinearly through the FWM process. Active controlling of the OIT can be achieved by varying a strong pump beam, and small frequency detuning of the pump can lead to a Fano-like asymmetric resonance to justify the interference nature of the OIT. Furthermore, the OIT observed here is a non-reciprocal one because the FWM gain is unidirectional owing to the conservation law of momentum. Our unique OIT scheme offers a completely new platform to study analogical atomic quantum interference effects in a simple manner. Moreover, it can be further exploited for critical on-chip photonics applications, such as optical isolator, all-optical switching and wavelength conversion.

\section{MATERIALS AND METHODS}

We developed an OIT window inside a single optical resonance dip of a whispering gallery mode (WGM) micro-cavity through a cavityenhanced FWM scheme, as shown in Figure 1. In the waveguidecoupled micro-cavity, light can tunnel from the input waveguide into

\footnotetext{
${ }^{1}$ The State Key Laboratory of Advanced Optical Communication Systems and Networks and Collaborative Innovation Center of IFSA, Department of Physics and Astronomy, Shanghai Jiao Tong University, Shanghai 200240, China; ${ }^{2}$ MOE Key Laboratory for Laser Plasmas, the University of Michigan-Shanghai Jiao Tong University Joint Institute, Shanghai Jiao Tong University, Shanghai 200240, China and ${ }^{3}$ Department of Mechanical Engineering, University of Michigan, Ann Arbor, MI 48109, USA Correspondence: W Wan, Email: wenjie.wan@sjtu.edu.cn; X Chen, Email: xfchen@sjtu.edu.cn

Received 28 September 2015; revised 7 January 2016; accepted 7 January 2016; accepted article preview online 14 January 2016
} 

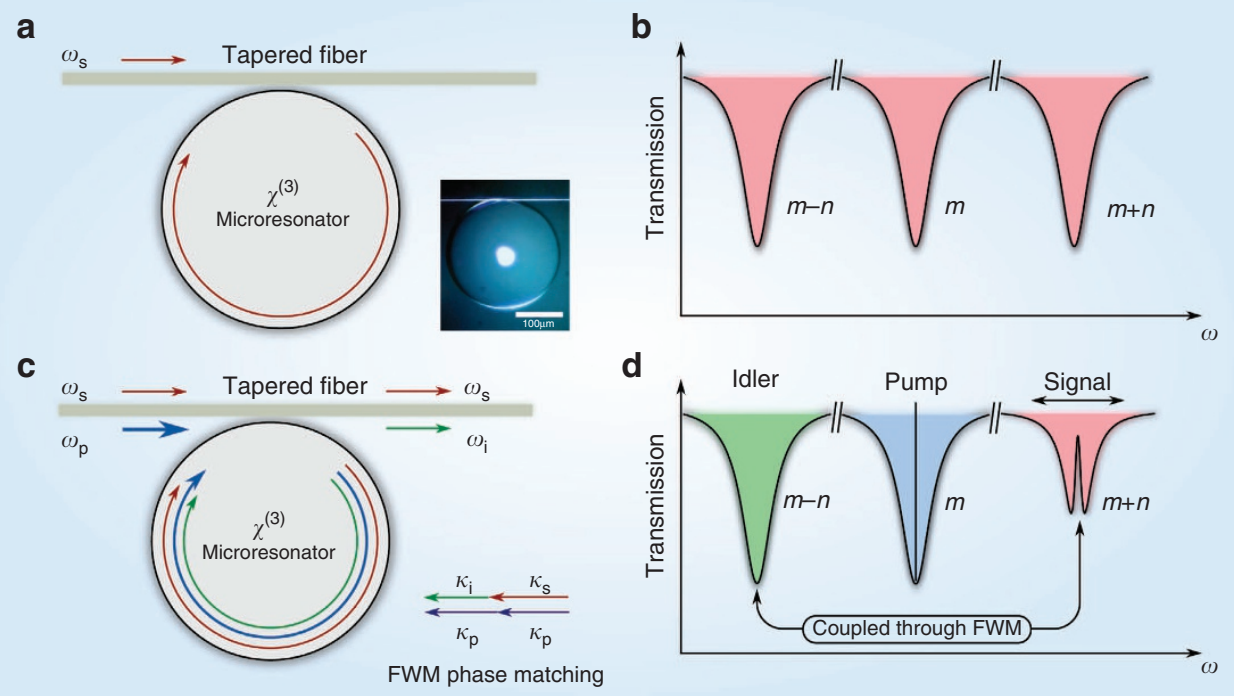

Figure 1 Schematic illustration of the optically induced transparency. (a) Light coupling into a micro-cavity through a tapered fiber at near-critical coupling conditions, providing (b) a transmission spectrum of the signal wave, $\omega_{\mathrm{s}}$. (c) Introducing a strong pump wave, $\omega_{\mathrm{p}}$, into the same micro-cavity and generating a nonlinear FWM by fulfilling the phase-matching condition. (d) A transparent window inside the transmission dip can be induced due to the nonlinear coupling of the two resonances at the signal and at the idler waves via the FWM process.

the micro-cavity to form the whispering gallery resonance mode by total internal reflection ${ }^{19}$. The resonance conditions lead to opaque dips in the transmission spectrum, as shown in Figure 1b, that is even perfectly opaque in critical coupling conditions ${ }^{20,21}$. Traditionally, such resonance line shapes cannot be modified unless the intrinsic properties of the cavity are altered, for example, size, surface scattering or absorption loss ${ }^{19}$. However, to obtain a transparency in an opaque resonance dip, that is, an EIT-like transmission spectrum, an external narrow gain/loss mechanism must be introduced. In atomics physics, this can be achieved by atomic resonant enhanced processes; however, in optics, cavity resonances facilitate the exploration of this narrow transparency, for example, using two coupled resonators with adjacent resonances ${ }^{10-12}$, double-layered resonators ${ }^{22}$ with close resonances or BS gain in an opto-mechanical micro-cavity ${ }^{15-18}$. However, these methods lack active all-optical control of the induced transparency. Furthermore, a hybrid approach, which combines ultra-slow light atomic resonance with photonic structured resonance, can also lead to induced transparency ${ }^{13}$. Again, however, this may still require cooling, vacuum systems and even a trapping mechanism to place the atoms within the photonic structure to maintain the atomic resonances.

Here we introduce a narrow parametric gain from a nonlinear degenerated FWM process to open up a transparency inside the opaque resonance dip. To achieve this, a strong pump beam is injected into one resonance at a frequency of $\omega_{m}$ ( $m$ is the azimuthal order of WGM modes) of a micro-cavity with $\chi^{(3)}$ nonlinearity, as shown in Figure 1c. This enables degenerated FWMs to be enhanced by the cavity resonances, providing parametric gains to two other distanced resonances that are symmetrically located at frequencies $\omega_{m+n}$ and $\omega_{m-n}$, which keeps $\omega_{m+n}+\omega_{m-n}=2 \omega_{m}$. Meanwhile, a second signal beam scans through the resonance near $\omega_{m+n}$, and a transparency peak can be obtained inside the original resonance dip, as shown in Figure 1d. The parametric gain is a narrow linewidth one owing to the cavity resonance's enhancement; hence, its linewidth is imprinted by the resonances. As explained below, for a given narrow linewidth (compared with the resonance's linewidth) pump, the transparency window inside the opaque dip of the signal transmission will copy the linewidth of the resonance near the idler wave's frequency, $\omega_{m-n}$.
Effectively, two resonances near the signal and idler waves' frequencies are nonlinearly coupled together via the FWM process. They interfere with each other, resulting in a hump-in-dip transmission spectrum. Note that, a broad-spectrum gain/loss-like scattering loss and absorption cannot lead to this OIT transmission, and it only affects the quality factor (Q-factor) of the resonance ${ }^{19}$. Unlike the previous works, for example, opto-mechanically induced transparency (OMIT) or BS-induced transparency $(\mathrm{BSIT})^{15-18}$, in which the narrow acoustic-induced gain requires careful tailoring both in the mechanical and optical modes to fulfill rigid energy and momentum conservations, our scheme does not require manipulating the two species of resonance modes and does not demand additional cooling or vacuum systems to stabilize those resonances. Hence, it can be ubiquitously attained in many resonant systems with $\chi^{(3)}$ nonlinearity.

To gain more physical insights, we now provide a precise discussion of the OIT theorem. For FWM in a resonant cavity system, its signal, $A_{\mathrm{s}}\left(\omega_{\mathrm{s}}\right)$, and idler, $A_{\mathrm{i}}\left(\omega_{\mathrm{i}}\right)$, beams can be coupled in a nonlinear fashion, which can be formulated in a set of coupled equations according to the coupled mode theory, as shown in Equations (1) and (2) (Supplementary Data). These cavity resonances are governed by the photon decay rate, $\kappa(\kappa=1 / \tau, \tau$ is the photon lifetime), including the internal rate, $\kappa_{\mathrm{O}}$ (due to internal absorption, scattering and radiation loss), and the external rate, $\kappa_{\mathrm{e}}$ (due to waveguide coupling), with a frequency detuning of $\Delta \omega$ from its central resonance of $\omega_{\mathrm{o}}$ for both the signal, $\omega_{\mathrm{s}}$, and the idler, $\omega_{\mathrm{i}}$ (s and i represent the signal and idler waves, respectively). The two frequency-detuning factors, $\Delta \omega_{\mathrm{s}}$ and $\Delta \omega_{\mathrm{i}}$, depend on each other through the FWM energy conservation: $\omega_{\mathrm{s}}$ $+\omega_{\mathrm{i}}=2 \omega_{\mathrm{p}}$, and they are on the scale of a single cavity resonance's linewidth $(\sim 1 / \tau)$. The most important coupling term, $g$, arises from nonlinear FWMs, effectively linking the two resonances of the signal and the idler together. In two coupled micro-cavities ${ }^{10-12}$, the linear coupling initiates EIT-like phenomena by interfering with two adjacent resonance modes. Although our approach of utilizing FWM to nonlinearly tie two separated resonances is genuinely different, only relevant works can be found on EIT from a multiple resonant atomic system $^{23}$. Even compared with FWM-based EIT in an atomic system, our OIT is achieved on a degenerated FWM scheme, and it only 
requires two inputs near $1550 \mathrm{~nm}$ telecommunication windows, which is very difficult to access with atomic systems:

$$
\begin{aligned}
\frac{\mathrm{d} A_{\mathrm{s}}\left(\omega_{\mathrm{s}}\right)}{\mathrm{d} t}= & \left(-\kappa_{\mathrm{os}}-\kappa_{\mathrm{es}}+\mathrm{i} \Delta \omega_{\mathrm{s}}\right) A_{\mathrm{s}}\left(\omega_{\mathrm{s}}\right)+\mathrm{i} \sqrt{2 \kappa_{\mathrm{es}}} A_{\mathrm{s}}^{\mathrm{in}}\left(\omega_{\mathrm{s}}\right) \\
& +\mathrm{i} g A_{\mathrm{i}}^{*}\left(\omega_{\mathrm{i}}\right) \\
\frac{\mathrm{d} A_{\mathrm{i}}\left(\omega_{\mathrm{i}}\right)}{\mathrm{d} t}= & \left(-\kappa_{\mathrm{oi}}-\kappa_{\mathrm{ei}}+\mathrm{i} \Delta \omega_{\mathrm{i}}\right) A_{\mathrm{i}}\left(\omega_{\mathrm{i}}\right)+\mathrm{i} g A_{\mathrm{s}}^{*}\left(\omega_{\mathrm{s}}\right)
\end{aligned}
$$

Under the assumption of FWM phase-matching conditions (Figure 1c), which can be obtained in a micro-cavity with small dispersion, the transmission rate of the signal (normalized with input) reads as:

$$
T_{\mathrm{s}}=\left(1+2 \frac{\kappa_{\mathrm{es}} X_{\mathrm{i}}^{*}}{X_{\mathrm{s}} X_{\mathrm{i}}^{*}-g^{2}}\right)^{2}
$$

where $X=-\kappa_{\mathrm{O}}-\kappa_{\mathrm{e}}+\mathrm{i} \Delta \omega$ (s and $\mathrm{i}$ represent signal and idler waves, respectively). The transmission $T_{\mathrm{S}}$ is affected by both resonances of the signal and idler. Moreover, it can be controlled by the nonlinear coupling term, $g$, which originated from the third-order Kerr nonlinearity (Supplementary Data). Because both the signal and idler waves are confined in the same micro-cavity and their wavelengths are close (few $\mathrm{nm}$ ), the decay rates of both resonances can be assumed to be the same. Furthermore, under the zero-detuning condition of $\Delta \omega_{\mathrm{s}}=-\Delta \omega_{\mathrm{i}}$, a transparency window can be observed in the $T_{\mathrm{s}}$ with proper $g$ factors, as shown in Figure $2 \mathrm{a}$, which provides the transmission spectra of the signal and the idler under different coupling strengths of $g$. As the nonlinear coupling strength gradually increases, the original opaque resonance dip first becomes shallow, then transits into a hump-in-dip spectrum and finally becomes an amplified peak above unity.

\section{RESULTS AND DISCUSSION}

The OIT arises from the interference of the signal and the idler resonances through nonlinear coupling during FWMs, in which the gain induced near the signal resonance is a narrow one enhanced by two other resonances at the pump and the idler. For a narrow linewidth pump (launched at one resonance of the micro-cavity), its FWM gain near the signal resonance purely imprints the idler resonance's linewidth through the nonlinear coupling, $g$, in Equations (1) and (2) to effectively create a virtual resonance peak near the signal resonance. This hump-in-dip spectrum near the signal resonance in Figure 2a results from the interference between the virtual gain peak and the original resonance dip. Meanwhile, a narrow gain peak, which grows with increasing $g$, is initiated simultaneously at the idler resonance in Figure $2 \mathrm{a}$, which verifies this cross-coupling. Unlike the EIT case, the dispersion relation during OIT is not significantly distorted because the widths of both resonances are relatively broad compared with the atomic resonance's linewidth in EIT or the BS gain's linewidth in OMIT. Hence, the slow light effect here can only be multiplied a few times (Supplementary Data), and the fast light effect is also absent in OIT. However, this may offer a unique opportunity for ultrafast optical switching applications, which requires a fast photon decay rate, that is, broad resonance ${ }^{24}$.

Experimentally, we verify the above theory by a tapered fibercoupled silica microsphere cavity, as shown in Figure 1, whose WGMs can be accessed by $1550 \mathrm{~nm}$ telecommunication lasers for both the pump and signal waves. The size of the microsphere is $\sim 250 \mu \mathrm{m}$, and it is carefully designed with weak anomalous dispersion to encourage nonlinear FWMs. A strong pump beam is injected into one of the resonances at $\sim 1548.52 \mathrm{~nm}$, and it is further locked to this resonance through a thermal self-locking mechanism ${ }^{25}$. When pumping above a threshold (several $\mathrm{mW}$ ), a stable optical frequency comb ${ }^{26,27}$ can be generated through FWMs, which offers us a reference to scan a second signal beam at one of the comb lines at $\sim 1535.64 \mathrm{~nm}$. Afterwards, we decrease the pump power under the threshold of the optical comb generation to observe the OIT. Figure $2 \mathrm{~b}-2 \mathrm{e}$ shows the experimental observations of the OIT spectra of the signal wave and their corresponding idler spectra under various pump intensities. Without the pump, the signal wave approaches a near-critical coupling situation, allowing only $<10 \%$ transmission at the center of the resonance. The linewidth of the resonance dip is $\sim 20 \mathrm{MHz}$, which corresponds to a Q-factor of $\sim 10^{7}$. After the injection of a weak pump at approximately $2 \mathrm{~mW}$, the spectrum opens up with an $\sim 30 \%$ transmission at the dip, and the linewidth shrinks to $\sim 15 \mathrm{MHz}$. Here, the third-order nonlinear susceptibility, $\chi^{(3)}$, of silica is $2.5 \times 10^{-22} \mathrm{~m}^{2} \mathrm{~V}^{-2}$, which corresponds to $g \approx 0.3$ in the numerical simulation (Figure 2a, Supplementary Data). When the pump intensity boosts to $\sim 3 \mathrm{~mW}$, a signature hump begins to exhibit itself inside the dip in Figure $2 \mathrm{~d}$. This hump spans $\sim 5 \mathrm{MHz}$, which is
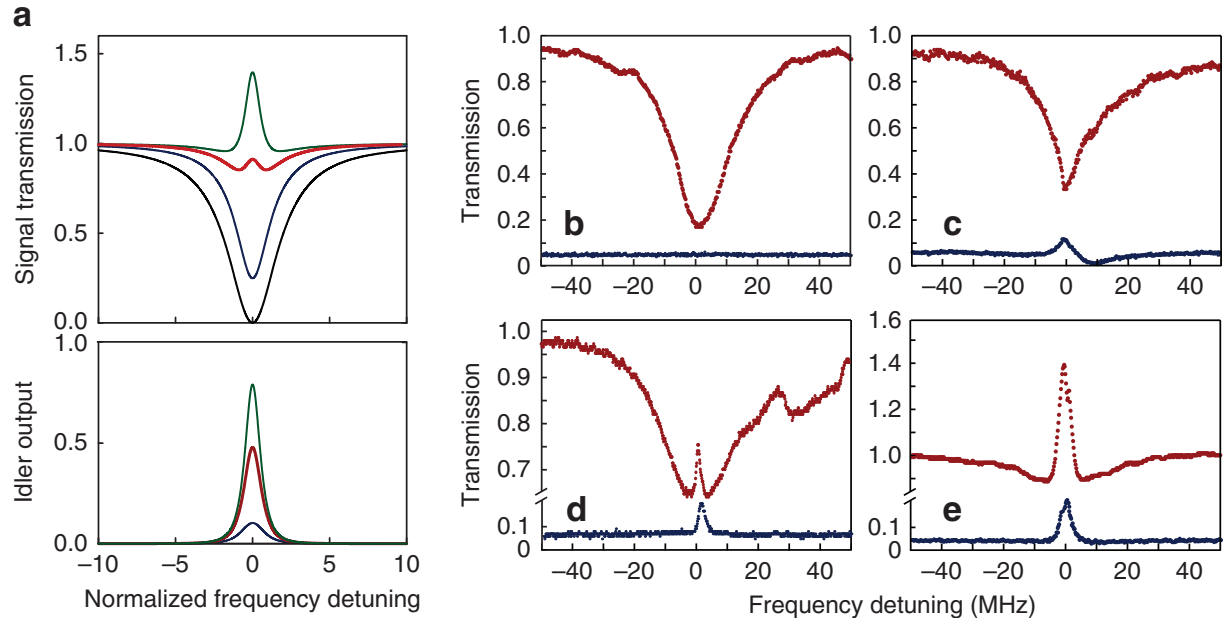

Figure 2 Theoretical and experimental transmission spectra of the signal and the idler under various nonlinear gain strengths for the zero-detuning condition of $\Delta \omega_{\mathrm{S}}=-\Delta \omega_{\mathrm{i}}$. (a) The theoretical results of the signal's transmission (upper) and the idler's generation coordinate (lower) with an increasing gain factor of $g=0,0.3,0.41$ and 0.45 for the black, blue, red and green curves, respectively. (b-e) Experimental measurements with increasing pump intensities of 0 , 2 , 3 and $5 \mathrm{~mW}$, respectively. The OIT manifests itself in d. 
broader than the transparency windows in EIT or OMIT. Finally, after the pump intensity reaches the threshold, an amplified peak replaces the original dip in Figure 2e. In addition, the idler waves' intensity grows coordinately with the increasing pumping. Moreover, if pumping harder, this process can be cascaded further to generate multiple amplified peaks at other resonances nearby to form an optical $\mathrm{comb}^{27}$. Notably, a small transmission jump manifests in the OIT spectra (Figure 2d), and this may be caused by interference with an adjacent higher order resonance mode to produce a Fano-like spectrum $^{22}$, which we discuss in the next section. In addition, the linewidth of OIT can be approximated by $\Gamma=\kappa \frac{g^{2}-\kappa^{2}}{g^{2}+\kappa^{2}}$ for $g>\kappa$ (above the OIT threshold, where $\kappa$ is the total decay rate for both signal \& idler resonances) or by $\Gamma=\kappa_{\kappa^{2}+g^{2}}$ for $g<\kappa$, (below the OIT threshold). This result explains the linewidth narrowing effect in Figure 2c, and the sharp OIT peak in Figure 2d (Supplementary Data).
Fano-like resonance in OIT

Interestingly, an asymmetric resonance in Figure 3 can be attained via the small detuning of two coupled resonances. This can be recognized as the Fano-Feshbach resonance ${ }^{28,29}$, which is known to originate from the interference of two neighboring resonances and provides an asymmetric profile of resonances. Here, we can create a non-zerodetuning scenario of $\Delta \omega_{\mathrm{s}} \neq-\Delta \omega_{\mathrm{i}}$, by either (1) setting $\omega_{\mathrm{so}}+\omega_{\mathrm{io}} \neq 2 \omega_{\mathrm{po}}$, which could be found for a non-phase-matched resonator, or by (2) detuning the frequency of the pump off its resonance center, $\omega_{\mathrm{po}}$, in a phase-matched scheme (Supplementary Data). Experimentally, we adapted the latter approach by slightly detuning the pump's frequency around the central resonance. Because the relationship of $\omega_{\mathrm{s}}+\omega_{\mathrm{i}}=2 \omega_{\mathrm{p}}$ and the phase-matching condition must be maintained, this effectively shifts the virtual resonance peak off the center of the signal resonance while pushing the idler gain peak to the other direction, as shown in
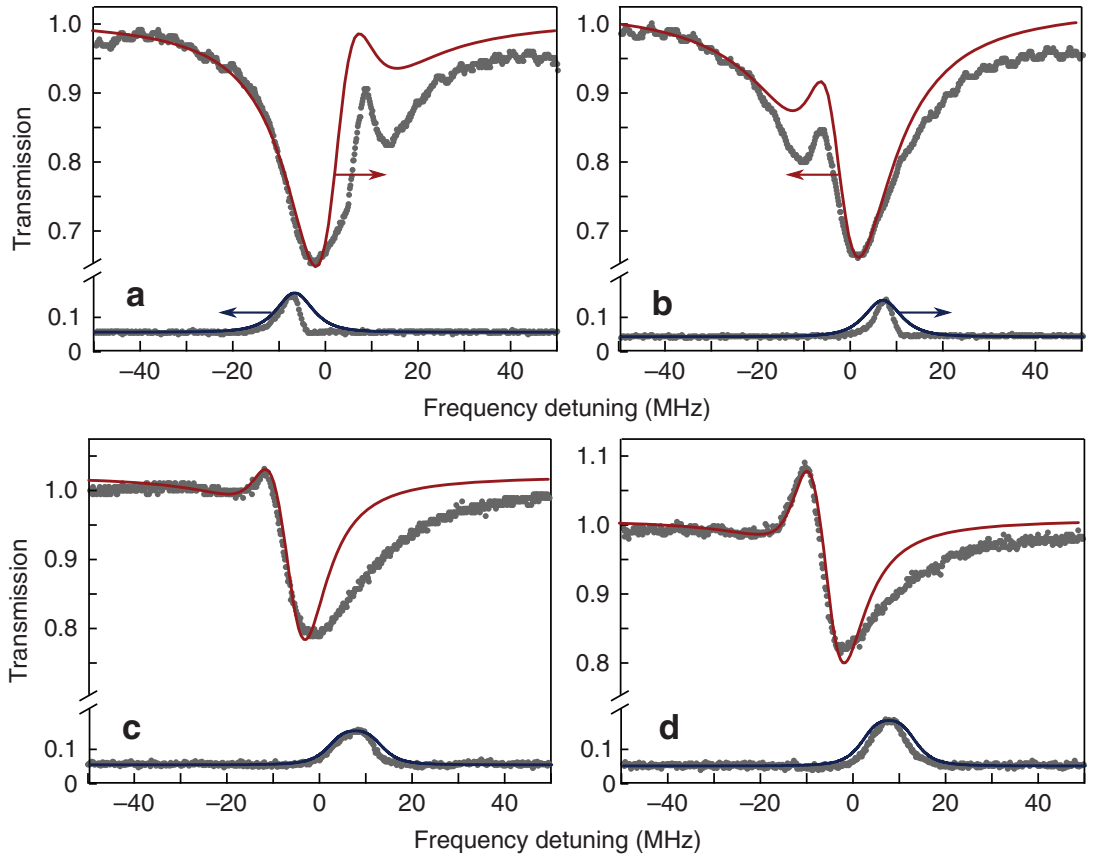

Figure 3 Fano-like resonance spectra for a non-zero-detuning condition. (a,b) asymmetric Fano-like resonance spectra by blue and red detuning of the pump. (c) Fano-like spectrum by further red detuning of the pump by increasing the gain in d. Dotted lines are measured experimental results, and the solid lines correspond to the calculations that are based on Equation (3). Upper curve: signal beam, lower curve: idler beam.

a

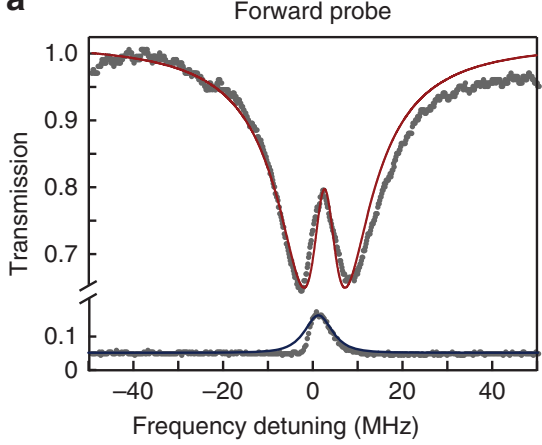

b

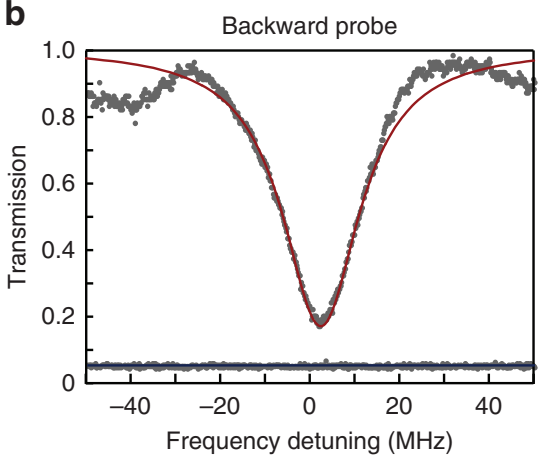

Figure 4 Demonstration of the non-reciprocal OIT. (a) The OIT is observed in the forward direction due to the unidirectional FWM gain. (b) The transparency window is absent in the backward direction. Dotted lines are the measured experimental results, and the solid lines correspond to the calculations that are based on Equation (3). Upper curve: signal beam, lower curve: idler beam. 
Figure 3. Ideally, the Fano resonance enables a constructive interference on one side and a destructive one on the other side due to a $\pi$-phase jump at the center of one resonance ${ }^{28,29}$. This is clearly pronounced by comparing the two OIT spectra in Figure $3 \mathrm{a}$ and $3 \mathrm{~b}$, when blue detuning or red detuning the pump at the location where the Fanolike resonance's symmetry flips. Further red detuning the pump pushes the OIT peak off to the edge, and it can be amplified by increasing the pump intensity. Attaining a Fano-like resonance in our case is allowed due to the similar linewidths of the two coupled resonances; however, in other situations in which the two resonances' linewidths significantly differ from each other, for example, OMIT or BSIT, such phenomenon is difficult to observe.

\section{Non-reciprocal transparency in OIT}

Finally, the observed OIT here is a non-reciprocal one, as shown in Figure 4. Clearly, the forward transmission is featured with the OIT window, which is absent in the backward transmission. The non-reciprocal distinction ratio can reach $\sim 6.5 \mathrm{~dB}$ at the center of the OIT window, which can be enlarged further to $\sim 26 \mathrm{~dB}$ under the critical coupling situation in which the backward transmission is minimized ${ }^{20}$. Such phenomena occurs because of the unidirectional gain nature of the FWM, which strictly follows the phase-matching conditions, in which the forward momentum carried by the pump only enables the FWM gain in the same direction while inhibiting the gain in reverse. In the microwave regime, a similar mechanism has been achieved in a non-reciprocal solid state superconducting qubits device ${ }^{30}$. However, in optics, a BSIT utilizing a non-reciprocal BS gain has recently been demonstrated. Our novel approach utilizes unidirectional FWM gain in a micro-cavity, which features a non-reciprocal gain arising from the strong pump that has no demand for the signal's input power (below $80 \mu \mathrm{W}$ ). Hence, our method may be favorable for solving the long-standing problem of non-magnetic optical isolation in on-chip photonic applications.

\section{CONCLUSIONS}

We have theoretically and experimentally demonstrated a new scheme of an optically induced transparency in a compact micro-cavity in an ambient environment. Because the OIT relies only on the nonlinearity and resonance properties of the medium, similar features should be ubiquitously expected among any other physical system with these characteristics. This system may offer a new avenue for a compact, integrated solution for all-optical processing and quantum information.

\section{CONFLICT OF INTEREST}

These authors declare no conflict of interest.

\section{AUTHOR CONTRIBUTIONS}

WW and YZ designed the study; YZ designed the experimental setup, performed the research and analyzed the data; WW and XC supervised the work; JY, ZS and JC helped with the fabrication; XL provided advice and helpful discussion; and YZ and WW wrote the paper. All authors reviewed the manuscript.

\section{ACKNOWLEDGEMENTS}

This work was supported by the National Natural Science Foundation of China (grant nos 11304201 and 61475100), the National 1000-plan Program (Youth), the Shanghai Pujiang Talent Program (grant no. 12PJ1404700) and the Shanghai Scientific Innovation Program (grant no. 14JC1402900).
1 Boller KJ, Imamoğlu A, Harris SE. Observation of electromagnetically induced transparency. Phys Rev Lett 1991; 66: 2593-2596.

2 Harris SE. Lasers without inversion: Interference of lifetime-broadened resonances. Phys Rev Lett 1989; 62: 1033-1036.

3 Kasapi A, Jain M, Yin GY, Harris SE. Electromagnetically induced transparency: propagation dynamics. Phys Rev Lett 1995; 74: 2447-2450.

4 Harris SE, Yamamoto Y. Photon switching by quantum interference. Phys Rev Lett 1998; 81: 3611-3614

5 Brunner D, Gerardot BD, Dalgarno PA, Wüst G, Karrai K et al. A coherent single-hole spin in a semiconductor. Science 2009; 325: 70-72.

6 Turukhin AV, Sudarshanam VS, Shahriar MS, Musser JA, Ham BS et al. Observation of ultraslow and stored light pulses in a solid. Phys Rev Lett 2001; 88: 023602.

7 Hau LV, Harris SE, Dutton Z, Behroozi CH. Light speed reduction to 17 metres per second in an ultracold atomic gas. Nature 1999; 397: 594-598.

8 Phillips DF, Fleischhauer A, Mair A, Walsworth RL, Lukin MD. Storage of light in atomic vapor. Phys Rev Lett 2001; 86: 783-786.

9 Liu C, Dutton Z, Behroozi CH, Hau LV. Observation of coherent optical information storage in an atomic medium using halted light pulses. Nature 2001; 409: 490-493.

10 Xu QF, Sandhu S, Povinelli ML, Shakya J, Fan SH et al. Experimental realization of an on-chip all-optical analogue to electromagnetically induced transparency. Phys Rev Lett 2006; 96: 123901.

11 Smith DD, Lepeshkin NN, Schweinsberg A, Gehring G, Boyd RW et al. Coupledresonator-induced transparency in a fiber system. Opt Commun 2006; 264: 163.

12 Yanik MF, Suh W, Wang Z, Fan SH. Stopping light in a waveguide with an all-optical analog of electromagnetically induced transparency. Phys Rev Lett 2004; 93: 233903.

13 Soljacic M, Lidorikis E, Joannopoulos JD, Hau LV. Electromagnetically induced transparency in microcavities. In: Taylor EW, editor. Proceedings of the Photonics for Space Environments IX. Denver, CO, USA: SPIE; 2004. 5554: p174.

14 Zhang S, Genov DA, Wang Y, Liu M, Zhang X. Plasmon-induced transparency in metamaterials. Phys Rev Lett 2008; 101: 047401.

15 Weis S, Rivière R, Deléglise S, Gavartin E, Arcizet 0 et al. Optomechanically induced transparency. Science 2010; 330: 1520-1523.

16 Safavi-Naeini AH, Alegre TPM, Chan J, Eichenfield M, Winger M et al. Electromagnetically induced transparency and slow light with optomechanics. Nature 2011; 472: 69-73.

17 Dong CH, Fiore V, Kuzyk MC, Wang HL. Optomechanical dark mode. Science 2012 ; 338: 1609-1613.

$18 \mathrm{Kim}$ J, Kuzyk MC, Han KW, Wang HL, Bahl G. Non-reciprocal Brillouin scattering induced transparency. Nat Physics 2015; 11: 275-280.

19 Chang RK, Campillo AJ. Optical Processes in Microcavities. Singapore: World Scientific; 1996.

20 Cai M, Painter O, Vahala KJ. Observation of critical coupling in a fiber taper to a silicamicrosphere whispering-gallery mode system. Phys Rev Lett 2000; 85: 74-77.

21 Wan WJ, Chong YD, Ge L, Noh H, Stone AD et al. Time-reversed lasing and interferometric control of absorption. Science 2011; 331: 889-892.

22 Xiao XF, He LN, Zhu JG, Yang L. Electromagnetically induced transparency-like effect in a single polydimethylsiloxane-coated silica microtoroid. Appl Phys Lett 2009; 94: 231115.

23 Zhang YP, Brown AW, Xiao M. Opening four-wave mixing and six-wave mixing channels via dual electromagnetically induced transparency windows. Phys Rev Lett 2007; 99: 123603.

24 Lipson M, Osgood JRM, Shin JH, Wada K. Introduction to the special issue on silicon photonics. IEEE J Sel Top Quantum Electron 2010; 16: 4-5.

25 Agha IH, Okawachi Y, Foster MA, Sharping JE, Gaeta AL. Four-wave-mixing parametric oscillations in dispersion-compensated high-Q silica microspheres. Phys Rev A 2007; 76: 043837.

26 Cundiff ST, Ye J. Colloquium: Femtosecond optical frequency combs. Rev Mod Phys 2003; 75: 325-342.

27 Kippenberg TJ, Holzwarth R, Diddams SA. Microresonator-based optical frequency combs. Science 2011; 332: 555-559.

28 Fano U. Sullo spettro di assorbimento dei gas nobili presso il limite dello spettro d'arco. Nuovo Cim 1935; 12: 154-161.

29 Feshbach H. Unified theory of nuclear reactions. Ann Phys 1958; 5: 357-390.

30 Kamal A, Clarke J, Devoret MH. Noiseless non-reciprocity in a parametric active device. Nat Physics 2011; 7: 311-315.

(c) (1) (2) This work is licensed under a Creative Commons AttributionNonCommercial-ShareAlike 4.0 International License. The images or other third party material in this article are included in the article's Creative Commons license, unless indicated otherwise in the credit line; if the material is not included under the Creative Commons license, users will need to obtain permission from the license holder to reproduce the material. To view a copy of this license, visit http://creative commons.org/licenses/by-nc-sa/4.0/ 